\title{
Projective Lag Synchronization and Parameter Identification of a New Hyperchaotic System
}

\author{
Wan-Li Guo Ming-Zhi Mao \\ School of Mathematics and Physics, China University of Geosciences, Wuhan 430074, China
}

\begin{abstract}
In this paper, the projective lag synchronization of a new hyperchaotic system with certain/uncertain parameters is addressed. Based on Lyapunov stability theory, a generic and simple controller is designed for the projective lag synchronization. Furthermore, with LaSalle's invariance principle, an adaptive method is proposed to identify the unknown parameters of the new hyperchaotic system based on the projective lag synchronization. Finally, numerical simulations are given to support the analytical approach.
\end{abstract}

Keywords: Projective lag synchronization, hyperchaotic system, parameter identification, Lyapunov stability theory, LaSalle's invariance principle.

\section{Introduction}

Many hyperchaotic systems, such as Lorenz hyperchaotic system $^{[1]}$, Chen hyperchaotic system ${ }^{[2]}$, Lü hyperchaotic system $^{[3]}$, the generalized Hénon-Heiles system ${ }^{[4]}$, have been extensively studied since the pioneering work of [5]. Hyperchaoic systems have at least two positive Lyapunov exponents. They have more complex behavior and abundant dynamics than a chaotic system. So they are more suitable for some engineering applications such as cryptography and secure communication. Hence, how to realize synchronization of hyperchaotic systems is an interesting and challenging task.

Since the notation of chaos synchronization was introduced by Pecora and Carroll ${ }^{[6]}$, it has been an active research topic and received extensive studies. It has many applications, e.g., chemical oscillators, population dynamics, physiological interactions, coupled neurons to mechanical oscillators, and lasers. Up to now, many effective control methods have been proposed to synchronize chaotic systems, e.g., generalized synchronization ${ }^{[7]}$, phase synchronization ${ }^{[8]}$, lag synchronization ${ }^{[9]}, \quad$ Q-S synchronization $^{[10]}$, projective synchronization ${ }^{[11]}, H_{\infty}$ synchronization $^{[12]}$, anticipating synchronization ${ }^{[13]}$, exponential synchronization ${ }^{[14]}$, etc. Among these regimes of synchronization, projective synchronization means that the corresponding state variables of drive-response hyperchaotic (chaotic) systems evolve in a proportion scale, and it includes the complete synchronization and the antisynchronization. On the other hand, the lag synchronization is more reasonable in our engineering applications, because time delay exists inevitably for finite signal transmission speeds or memory effects in communication.

Therefore, based on these two points, we firstly proposed the notation of projective lag synchronization which includes the projective synchronization and the lag syn-

\footnotetext{
Manuscript received August 30, 2012; revised November 7, 2012

This work was supported by National Basic Research Program of China (973 program) (No. 2011CB710605), TianYuan Special Funds of National Natural Science Foundation of China (No. 11226134), National Natural Science Foundation of China (No.61273215)
}

chronization at the same time. It is more meaningful because it has a more general synchronization form and can suit for more real cases. Moreover, in practical situations, there exist many cases with uncertain information, e.g., uncertain system parameters may destroy the synchronization. Therefore, how to effectively synchronize two hyperchaotic systems with unknown parameters is an important problem for theoretical research and practical applications. Parameter estimations of single or coupled nonlinear system(s) based on the complete synchronization method have been investigated ${ }^{[15-19]}$. In this paper, we will propose a novel method to identify the unknown parameters based on the projective lag synchronization of a new hyperchaotic system $^{[20]}$, and introduce a key condition that uncertain parameters can be identified correctly when projective lag synchronization is achieved.

This paper is organized as follows. The hyperchaotic system is introduced in Section 2. The projective lag synchronization and parameter identification of the hyperchaotic system are discussed in Sections 3 and 4, respectively. Numerical simulations are given in Section 5. Finally, the paper is concluded in Section 6.

\section{System description}

Recently, a new four-dimensional continuous autonomous hyperchaotic system ${ }^{[20]}$ was constructed as

$$
\left\{\begin{array}{l}
\dot{x}_{1}=a\left(x_{2}-x_{1}\right)+x_{4} \\
\dot{x}_{2}=x_{1} x_{3}-x_{2} \\
\dot{x}_{3}=b-x_{1} x_{2}-c x_{3} \\
\dot{x}_{4}=d x_{4}-x_{2} x_{3}
\end{array}\right.
$$

where $x_{1}, x_{2}, x_{3}$ and $x_{4}$ are state variables, and $a, b, c$ and $d$ are real constant parameters. After $5 \times 10^{4}$ iterations, we have four Lyapunov exponents which are 1.400240, $0.313208,-0,968585$ and -3.327758 when $a=5, b=$ $16, c=1$ and $d=-0.5$. It implies that there exists a hyperchaotic attractor ${ }^{[20]}$. The three-dimensional phase portrait of the hyperchaotic attractor is illustrated in Fig. 1. 

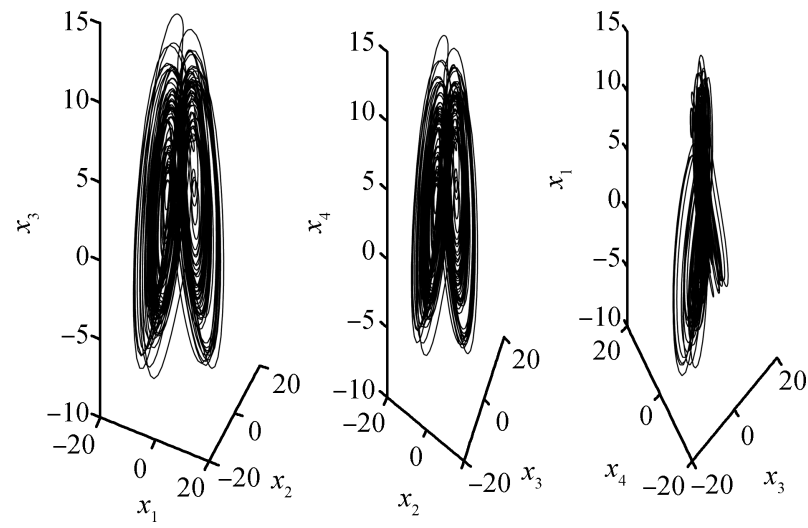

Fig. 1 Hyperchaotic attractor

\subsection{Projective lag synchronization of the new hyperchaotic system}

For the drive hyperchaotic system (1), the response system can be described as

$$
\left\{\begin{array}{l}
\dot{y}_{1}=a\left(y_{2}-y_{1}\right)+y_{4} \\
\dot{y}_{2}=y_{1} y_{3}-y_{2}+u_{1} \\
\dot{y}_{3}=b-y_{1} y_{2}-c y_{3}+u_{2} \\
\dot{y}_{4}=d y_{4}-y_{2} y_{3}+u_{3}
\end{array}\right.
$$

where $u_{1}, u_{2}$ and $u_{3}$ are nonlinear controllers to be designed so that systems (1) and (2) can be synchronized.

Let the error variables be

$$
\left\{\begin{array}{l}
e_{1}=y_{1}-\lambda x_{1}(t-\tau) \\
e_{2}=y_{2}-\lambda x_{2}(t-\tau) \\
e_{3}=y_{3}-\lambda x_{3}(t-\tau) \\
e_{4}=y_{4}-\lambda x_{4}(t-\tau)
\end{array}\right.
$$

where $\lambda \neq 0$ is the proportion scale, and $\tau$ is constant, representing the time delay or lag.

Substituting (1) and (2) into (3), we have the following error dynamical system:

$$
\left\{\begin{aligned}
\dot{e}_{1}= & -a e_{1}+a e_{2}+e_{4} \\
\dot{e}_{2}= & -e_{2}+y_{1} y_{3}-\lambda x_{1}(t-\tau) x_{3}(t-\tau)+u_{1} \\
\dot{e}_{3}= & -c e_{3}-y_{1} y_{2}+\lambda x_{1}(t-\tau) x_{2}(t-\tau)+ \\
& (1-\lambda) b+u_{2} \\
\dot{e}_{4}= & d e_{4}-y_{2} y_{3}+\lambda x_{2}(t-\tau) x_{3}(t-\tau)+u_{3} .
\end{aligned}\right.
$$

Definition 1. The drive system (1) and the response system (2) are said to achieve projective lag synchronization, if the solution of the error dynamical system (4) satisfies $\lim _{t \rightarrow \infty} e_{i}=0(i=1,2,3,4)$ for arbitrary initial conditions $x(0)=\left(x_{1}(0), x_{2}(0), x_{3}(0), x_{4}(0)\right)^{\mathrm{T}}$ and $y(0)=$ $\left(y_{1}(0), y_{2}(0), y_{3}(0), y_{4}(0)\right)^{\mathrm{T}}$.

Specially, for $\tau=0$, it is called the projective synchronization. For $\lambda=1$, it is called the lag synchronization.

Theorem 1. Systems (1) and (2) will approach exponential projective lag synchronization for any initial condition with the following control law:

$$
\left\{\begin{array}{l}
u_{1}=-y_{1} y_{3}+\lambda x_{1}(t-\tau) x_{3}(t-\tau)-a e_{1} \\
u_{2}=y_{1} y_{2}-\lambda x_{1}(t-\tau) x_{2}(t-\tau)+(\lambda-1) b \\
u_{3}=y_{2} y_{3}-\lambda x_{2}(t-\tau) x_{3}(t-\tau)-e_{1}
\end{array}\right.
$$

Proof. Construct a Lyapunov function

$$
V(t)=\frac{1}{2}\left(e_{1}^{2}+e_{2}^{2}+e_{3}^{2}+e_{4}^{2}\right) .
$$

With (5), the time derivative of the Lyapunov function along the trajectories of system (4) is

$$
\begin{aligned}
& \dot{V}(t)= e_{1} \dot{e}_{1}+e_{2} \dot{e}_{2}+e_{3} \dot{e}_{3}+e_{4} \dot{e}_{4}= \\
& e_{1}\left(-a e_{1}+a e_{2}+e_{4}\right)+ \\
& e_{2}\left(-e_{2}+y_{1} y_{3}-\lambda x_{1}(t-\tau) x_{3}(t-\tau)+u_{1}\right)+ \\
& e_{3}\left(-c e_{3}-y_{1} y_{2}+\lambda x_{1}(t-\tau) x_{2}(t-\tau)+\right. \\
&\left.(1-\lambda) b+u_{2}\right)+ \\
& e_{4}\left(y_{2} y_{3}-\lambda x_{2}(t-\tau) x_{3}(t-\tau)-e_{1}\right)= \\
&-a e_{1}^{2}-e_{2}^{2}-c e_{3}^{2}+d e_{4}^{2} .
\end{aligned}
$$

Let $\eta=\min (a, 1, c,-d)$, then we have

$$
\dot{V}(t) \leqslant-\eta V(t)
$$

which yields

$$
V(t) \leqslant V(0) \mathrm{e}^{-2 \eta t}
$$

This implies that $\lim _{t \rightarrow \infty} e_{i}=0(i=1,2,3,4)$, which guarantees the global and exponential asymptotical stability of the origin of system (4). In other word, systems (1) and (2) achieve global and exponential projective lag synchronization.

Remark 1. If $\tau=0$, then systems (1) and (2) asymptotically achieve projective synchronization. If $\lambda=1$, then systems (1) and (2) asymptotically achieve lag synchronization.

\section{Parameter identification based on projective lag synchronization}

In this section, suppose parameters $a, b, c$ and $d$ are unknown in the hyperchaotic system (1). We will focus on identifying the unknown parameters via using the projective lag synchronization theory and adaptive control techniques.

The response system is described by

$$
\left\{\begin{array}{l}
\dot{y}_{1}=\hat{a}\left(y_{2}-y_{1}\right)+y_{4} \\
\dot{y}_{2}=y_{1} y_{3}-y_{2}+u_{1} \\
\dot{y}_{3}=\hat{b}-y_{1} y_{2}-\hat{c} y_{3}+u_{2} \\
\dot{y}_{4}=\hat{d} y_{4}-y_{2} y_{3}+u_{3}
\end{array}\right.
$$

where $\hat{a}, \hat{b}, \hat{c}$ and $\hat{d}$ are the estimations of the uncertain parameters $a, b, c$ and $d$, and $u_{i}(i=1,2,3)$ are the controllers such that the hyperchaotic systems get projective lag synchronization.

Denote $\tilde{a}=\hat{a}-a, \tilde{b}=\hat{b}-b, \tilde{c}=\hat{c}-c$ and $\tilde{d}=\hat{d}-$ d. Substituting (1) and (5) into (3), we have the error dynamical system as

$$
\left\{\begin{aligned}
\dot{e}_{1}= & -a e_{1}+a e_{2}+\tilde{a}\left(y_{2}-y_{1}\right)+e_{4} \\
\dot{e}_{2}= & -e_{2}+y_{1} y_{3}-\lambda x_{1}(t-\tau) x_{3}(t-\tau)+u_{1} \\
\dot{e}_{3}= & -c e_{3}+\tilde{b}+(1-\lambda) b-y_{1} y_{2}+ \\
& \lambda x_{1}(t-\tau) x_{2}(t-\tau)-\tilde{c} y_{3}+u_{2} \\
\dot{e}_{4}= & d e_{4}+\tilde{d} y_{4}-y_{2} y_{3}+\lambda x_{2}(t-\tau) x_{3}(t-\tau)+u_{3} .
\end{aligned}\right.
$$


Theorem 2. We select the controllers in (6) as

$$
\left\{\begin{array}{l}
u_{1}=-y_{1} y_{3}+\lambda x_{1}(t-\tau) x_{3}(t-\tau)-a e_{1} \\
u_{2}=y_{1} y_{2}-\lambda x_{1}(t-\tau) x_{2}(t-\tau)+(\lambda-1) b \\
u_{3}=y_{2} y_{3}-\lambda x_{2}(t-\tau) x_{3}(t-\tau)-e_{1} .
\end{array}\right.
$$

And we use the following updating laws as

$$
\left\{\begin{array}{l}
\dot{\hat{a}}=-e_{1}\left(y_{2}-y_{1}\right) \\
\dot{\hat{b}}=-e_{3} \\
\dot{\hat{c}}=e_{3} y_{3} \\
\dot{\hat{d}}=-e_{4} y_{4} .
\end{array}\right.
$$

Then, systems (1) and (6) achieve projective lag synchronization. And the uncertain parameters are well estimated from the system parameters $a, b, c$ and $d$ in the sense that $\lim _{t \rightarrow \infty} \hat{a}=a, \lim _{t \rightarrow \infty} \hat{b}=b, \lim _{t \rightarrow \infty} \hat{c}=c$ and $\lim _{t \rightarrow \infty} \hat{d}=$ d.

Proof. Choose the following Lyapunov functional candidate:

$$
V(t)=\frac{1}{2}\left(e_{1}^{2}+e_{2}^{2}+e_{3}^{2}+e_{4}^{2}+\tilde{a}^{2}+\tilde{b}^{2}+\tilde{c}^{2}+\tilde{d}^{2}\right) .
$$

Differentiating $V(t)$ with respect to time along the solution of (7) yields

$$
\begin{aligned}
\dot{V}(t)= & e_{1}^{2}+e_{2}^{2}+e_{3}^{2}+e_{4}^{2}+\tilde{a}^{2}+\tilde{b}^{2}+\tilde{c}^{2}+\tilde{d}^{2}= \\
& e_{1} \dot{e}_{1}+e_{2} \dot{e}_{2}+e_{3} \dot{e}_{3}+e_{4} \dot{e}_{4}+\tilde{a} \dot{\tilde{a}}+\tilde{b} \dot{\tilde{b}}+\tilde{c} \dot{\tilde{c}}+\tilde{d} \dot{\tilde{d}}= \\
& e_{1}\left(-a e_{1}+a e_{2}+\tilde{a}\left(y_{2}-y_{1}\right)+e_{4}\right)+ \\
& e_{2}\left(-e_{2}+y_{1} y_{3}-\lambda x_{1}(t-\tau) x_{3}(t-\tau)+u_{1}\right)+ \\
& e_{3}\left(-c e_{3}+\tilde{b}+(1-\lambda) b-y_{1} y_{2}+\right. \\
& \left.\lambda x_{1}(t-\tau) x_{2}(t-\tau)-\tilde{c} y_{3}+u_{2}\right)+ \\
& e_{4}\left(d e_{4}+\tilde{d} y_{4}-y_{2} y_{3}+\lambda x_{2}(t-\tau) x_{3}(t-\tau)+u_{3}\right)- \\
& \tilde{a} e_{1}\left(y_{2}-y_{1}\right)-\tilde{b} e_{3}+\tilde{c} e_{3} y_{3}-\tilde{d} e_{4} y_{4}= \\
& -a e_{1}^{2}-e_{2}^{2}-c e_{3}^{2}+d e_{4}^{2}
\end{aligned}
$$

Obviously, $\dot{V}=0$ if and only if $e_{i}=0, i=1,2,3,4$, thus $E=\{\dot{V}=0\}=\{e(t)=0\}$. So set $M=\{e(t)=$ $0, \hat{a}=A, \hat{b}=b, \hat{c}=c, \hat{d}=d\}$ is the largest invariant set of set $E$ for the error system (7). According to LaSalle's invariance principle ${ }^{[1]}$, starting with arbitrary initial values, the trajectory of error system (7) asymptotically converges to set $M$, i.e., $e_{i} \rightarrow 0, \hat{a} \rightarrow a, \hat{b} \rightarrow b, \hat{c} \rightarrow c$ and $\hat{d} \rightarrow d$ as $t \rightarrow \infty$. This implies that these unknown parameters can be successfully estimated using the adaptive feedback method proposed in Theorem 2.

\section{Numerical simulation}

In this section, we will show the numerical results for projective lag synchronization and parameter identification of the new hyperchaotic system to verify and illustrate the effectiveness of the theoretical analysis in Sections 3 and 4 .

\subsection{Projective lag synchronization of the new hyperchaotic system}

In this subsection, we set $a=5, b=16, c=1$ and $d=$ -0.5 in system (1). By the control method (5), one has the projective lag synchronization of systems (1) and (2).

Fig. 2 displays the trajectories of systems (1) and (2) when $\lambda=2$ and $\tau=1$. Fig. 3 shows the trajectories of systems (1) and (2) when $\lambda=-1$ and $\tau=1$. From these two figures, we can see that systems (1) and (2) get projective lag synchronization.
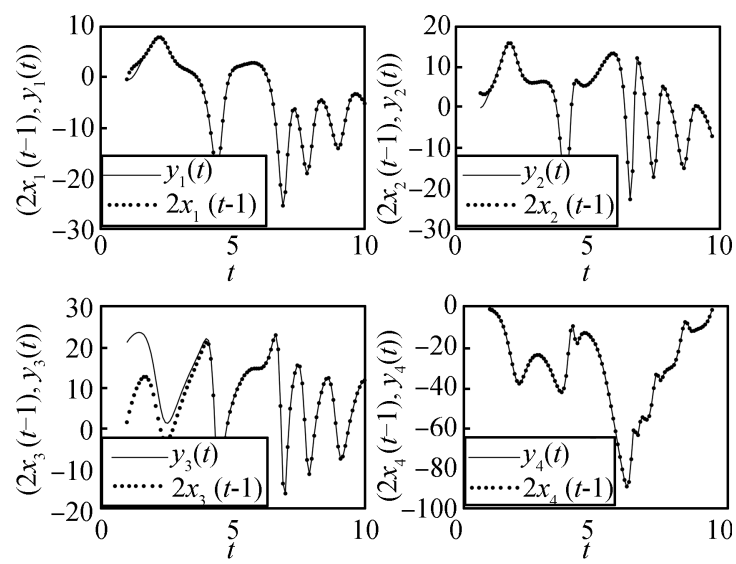

Fig. 2 The trajectories of systems (1) and (2) when $\lambda=2$ and $\tau=1$
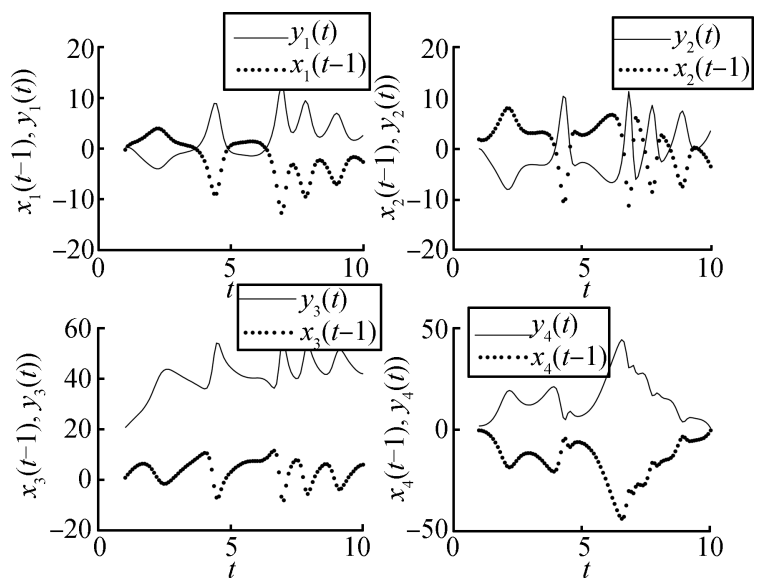

Fig. 3 The trajectories of systems (1) and (2) when $\lambda=-1$ and $\tau=1$

\subsection{Parameter identification based on pro- jective lag synchronization}

In this subsection, we also set $a=5, b=16, c=1$ and $d=-0.5$ in system (1). By the updating laws (8) and (9), the numerical results are shown in Figs. 4 and 5. It can be seen that the drive system (1) and the response system (6) get projective lag synchronization, and at the same time, unknown parameters $a, b, c$ and $d$ are identified correctly.

\section{Conclusions}

In this paper, the projective lag synchronization of a new hyperchaotic system with certain/uncertain parameters was addressed. Based on the Lyapunov stability theory, a generic and simple controller was designed for the projective lag synchronization. Moreover, with the LaSalle's invariance principle, an adaptive method was proposed to identify the unknown parameters of the new hyperchaotic system based on projective lag synchronization. Finally, numerical simulations fully supported the analytical approach. 

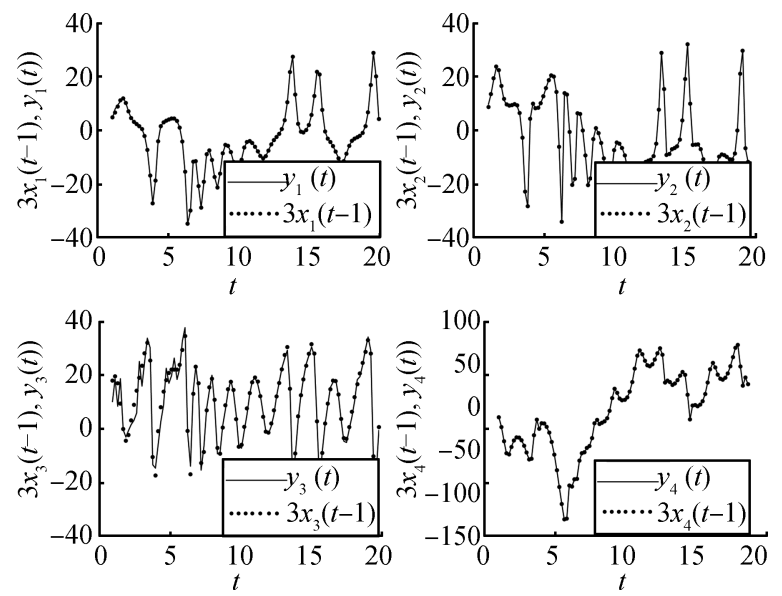

Fig. 4 The trajectories of systems (1) and (6) when $\lambda=3$ and $\tau=0.5$

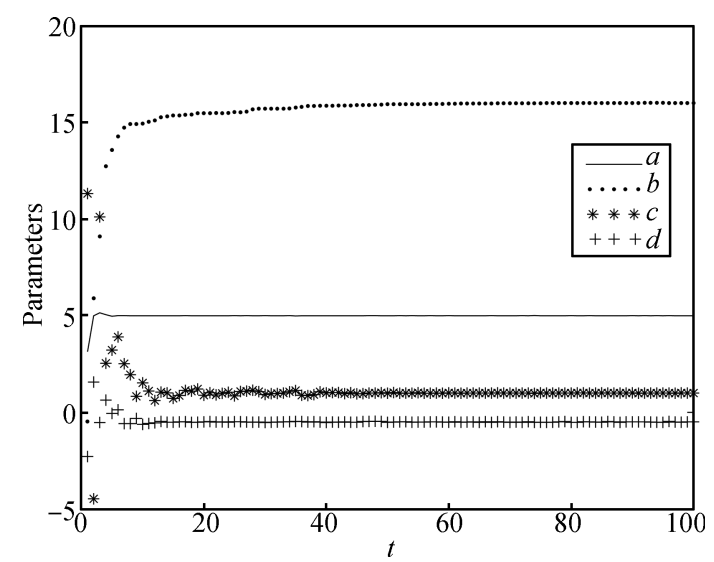

Fig. 5 Parameter identification of system (6)

\section{Acknowledgement}

The authors thank the referees and the editors for their valuable comments and suggestions on the improvement of this paper.

\section{References}

[1] T. G. Gao, G. R. Chen, Z. Q. Chen, S. J. Cang. The generation and circuit implementation of a new hyper-chaos based upon Lorenz system. Physics Letters A, vol.361, no. 1-2, pp. 78-86, 2007.

[2] Y. X. Li, W. K. S. Tang, G. R. Chen. Generating hyperchaos via state feedback control. International Journal of Bifurcation and Chaos, vol. 15, no. 10, pp. 3367-3375, 2005.

[3] A. M. Chen, J. N. Lu, J. H. Lü, S. M. Yu. Generating hyperchaotic Lü attractor via state feedback control. Physica A: Statistical Mechanics and its Applications, vol. 364, pp. 103-110, 2006.

[4] A. Jayaram, M. Tadi. Synchronization of chaotic systems based on SDRE method. Chaos, Solitons \& Fractals, vol. 28, no. 3, pp. 707-715, 2006.

[5] O. E. Rössler. An equation for hyperchaos. Physics Letters A, vol. 71, no. 2-3, pp. 155-157, 1979.

[6] L. M. Pecora, T. L. Carroll. Synchronization in chaotic systems. Physical Review Letters, vol.64, no. 8, pp. 821-824, 1990.

[7] L. Kocarev, U. Parlitz. Generalized synchronization, predictability, and equivalence of unidirectionally coupled dynamical systems. Physical Review Letters, vol. 76, no. 11, pp. 1816-1819, 1996.
[8] U. E. Vincent, A. N. Njah, O. Akinlade, A. R. T. Solarin. Phase synchronization in unidirectionally coupled chaotic ratchets. Chaos: An Interdisciplinary Journal of Nonlinear Science, vol. 14, no. 4, pp. 1018-1025, 2004.

[9] M. G. Rosenblum, A. S. Pikovsky, J. Kurths. From phase to lag synchronization in coupled chaotic oscillators. Physical Review Letters, vol. 78, no. 22, pp. 4193-4196, 1997.

[10] Z. Y. Yan. Q-S (lag or anticipated) synchronization backstepping scheme in a class of continuous-time hyperchaotic systems - a symbolic-numeric computation approach. Chaos: An Interdisciplinary Journal of Nonlinear Science, vol. 15, no. 2, 023902, 2005.

[11] Q. J. Zhang, J. A. Lu, Z. Jia. Global exponential projective synchronization and lag synchronization of hyperchaotic Lü system. Communications in Theoretical Physics, vol. 51, no. 4, pp. 679-683, 2009.

[12] J. H. Park, D. H. Ji, S. C. Won, S. M. Lee. $H_{\infty}$ synchronization of time-delayed chaotic systems. Applied Mathematics and Computation, vol. 204, no. 1, pp. 170-177, 2008.

[13] H. U. Voss. Anticipating chaotic synchronization. Physical Review E, vol. 61, no. 5, pp. 5115-5119, 2000.

[14] J. H. Park. Exponential synchronization of the Genesio-Tesi chaotic system via a novel feedback control. Physica Scripta vol. 76 , no. 6 , pp. 617-622, 2007.

[15] H. G. Zhang, H. Wei, Z. L. Wang, T. Y. Chai. Adaptive synchronization between two different chaotic systems with unknown parameters. Physics Letters A, vol. 350, pp. 363366, 2006.

[16] J. H. Park. Adaptive controller design for modified projective synchronization of Genesio-Tesi chaotic system with uncertain parameters. Chaos, Solitons \& Fractals, vol. 34, no. 4, pp. 1154-1159, 2007.

[17] J. H. Park, S. M. Lee, O. M. Kwon. Adaptive synchronization of Genesio-Tesi chaotic system via a novel feedback control. Physics Letters A, vol. 371, no. 4, pp. 263-270, 2007.

[18] M. T. Yassen. Adaptive chaos control and synchronization for uncertain new chaotic dynamical system. Physics Letters A, vol. 350, no. 1-2, pp. 36-43, 2006.

[19] S. Bowong. Adaptive synchronization of chaotic systems with unknown bounded uncertainties via backstepping approach. Nonlinear Dynamics, vol.49, no.1-2, pp.59-70, 2007.

[20] X. F. Li, A. C. Leung, X. J. Liu, X. P. Han, Y. D. Chu Adaptive synchronization of identical chaotic and hyperchaotic systems with uncertain parameters. Nonlinear Analysis: Real World Applications, vol. 11, no. 4, pp. 2215-2223, 2010.

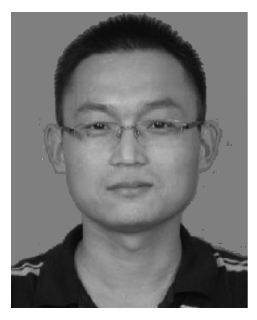

Wan-Li Guo graduated from Qingdao University, China in 2005. He received the Ph. D. degree from Wuhan University, China in 2010. He is currently a lecturer at School of Mathematics and Physics, China University of Geosciences (Wuhan), China.

His research interests include dynamic systems and automatic control.

E-mail: guowanliff@163.com (Corresponding author)

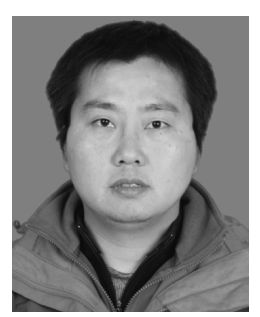

Ming-Zhi Mao received his Ph. D. degree from Shanghai Jiao Tong University in 2009. Since 2012, he has been an associate professor at China University of Geosciences, China. At present, he is also supported by the China Postdoctoral Science Foundation.

His research interests include stochastic process, probability, and statistics.

E-mail: mingzhi-mao@163.com 